\title{
A survey on hand hygiene practice among anaesthetists
}

\author{
K Rupasingha ${ }^{1}$, N Karunarathne ${ }^{2}$ \\ Registrar in Anaesthesiology ${ }^{l}$, National Hospital Sri Lanka, Colombo, Sri Lanka. Consultant \\ Anaesthetist ${ }^{2}$, Sri Jayawardenapura General Hospital, Kotte, Sri Lanka.
}

*Corresponding author: kishan_rupasingha@yahoo.com

\begin{abstract}
Background
The current prevalence of health care associated infections (HCAIs) is a major public health concern. Hand-mediated transmission is the major contributory factor to HCAIs and the intra operative environment serves as a risk factor for the development of hospital acquired infections. Failure to wear gloves and effective hand washing on patient contact are particular problems in the practice of anaesthesia where there is a high incidence of exposure to patient blood and saliva.
\end{abstract}

\begin{abstract}
Materials and Method
Data was collected through a two page questionnaire from 130 anaesthetists in National Hospital of Sri Lanka irrespective of the seniority or the position. Duly filled sheets were collected after one month and analyzed through SPSS 13 to determine the level of adherence.

Results

130 questionnaires were distributed and 100 received duly completed. $63 \%$ of participants were from general surgical section, $22 \%$ from neurosurgical section and the rest were from the cardiothoracic section.In general $25 \%$ of the female participants always wore gloves before handling each patient. $61 \%$ from both males and females confirmed wearing gloves only sometimes. $90 \%$ of the female participants always wore gloves before inserting NG tubes whereas only $70 \%$ of males did so.It was found that only $6.6 \%$ of the participants wore gloves when performing i.v. cannulation in cardiothoracic practice. $53 \%$ of the cardiothoracic anaesthetists never wore gloves when performing i.v. cannulation.
\end{abstract}

\section{Conclusion and Recommendations}

It was revealed that hand hygiene practices among anaesthetists are not at the satisfactory level and it is recommended to form a proper institutional guideline in infection control in anaesthetic practice. Further it is also recommended to conduct general awareness programs regularly and make hand hygiene a routine practice. Also a gradual culture change is recommended within theatres with guidance in adhering to proper hand hygiene practices.

Keywords: hand hygiene; contamination; health care associated infections; prevention

\section{Introduction}

Effective hand hygiene and correct use of gloves before every episode of direct patient contact will result in a significant reduction in the transfer of potential pathogens and a decrease in the incidence of preventable health care associated infections. Anaesthetists must ensure that good hand hygiene becomes an indispensible part of their clinical practice. Precautions aimed at preventing the transmission of organisms between patient and anaesthetist or between patients must be a routine part of anaesthetic practice. Objective of this study is to ascertain adequacy of current practices of anaesthetists in relation to infection control as they have an important role in preventing nosocomial infection.

Anaesthetists are involved in the care of patients who may harbor potentially pathogenic organisms, which may not be obvious or readily identifiable. All blood 
and body fluids, substances, secretions, and excretions may be considered to be potentially infective regardless of the perceived risk of the source. Hand mediated transmission is the major contributing factor to infection associated with health care. Further incorrect use of gloves and poor hand decontamination could actually spread infection between patients. ${ }^{1}$

\section{Material and methods}

Data was collected through a two page questionnaire from 130 anaesthetists in National Hospital of Sri Lanka. Questionnaire was developed based on an extensive literature review and guidelines used were AAGBI safety guidelines published by the Association of Anaesthetists of Great Britain and Ireland ${ }^{2}$. The questionnaires were distributed among anaesthetists and duly filled sheets were collected after one month and analyzed with SPSS 13.

\section{Results}

The response rate was $76 \%$.

Table 1 Data collection according to subspecialties

\begin{tabular}{|l|c|}
\hline \multicolumn{1}{|c|}{ Section } & Percentage \\
\hline Neurosurgical & $22 \%$ \\
\hline Cardiothoracic & $15 \%$ \\
\hline General Surgical & $63 \%$ \\
\hline \multicolumn{1}{|c|}{ Total } & $\mathbf{1 0 0 \%}$ \\
\hline
\end{tabular}

Table 2 Number of years' experience in anaesthesia

\begin{tabular}{|l|c|}
\hline $\begin{array}{c}\text { Years of experience } \\
\text { in Anaesthesia }\end{array}$ & $\%$ \\
\hline$<1$ Year & $1 \%$ \\
\hline$>1$ Year $<5$ Years & $29 \%$ \\
\hline $5-10$ Years & $57 \%$ \\
\hline$>10$ Years & $13 \%$ \\
\hline
\end{tabular}

$41 \%$ of the participants were females and $61 \%$ were medical officers.
Table 3 Designations and gender

\begin{tabular}{|l|c|}
\hline Designation & $\%$ \\
\hline MO & $61 \%$ \\
\hline Registrar & $22 \%$ \\
\hline Senior Registrar & $17 \%$ \\
\hline Total & $\mathbf{1 0 0 \%}$ \\
\hline Gender & $\mathbf{\%}$ \\
\hline Male & $41 \%$ \\
\hline Female & $59 \%$ \\
\hline Total & $\mathbf{1 0 0 \%}$ \\
\hline
\end{tabular}

Hand hygiene practice was poor in males with more than 10 years anaesthetic experience but the increase of the experience showed positivity towards hand hygiene and correct use of gloves in the females. In general $25 \%$ of the female participants always wore gloves before handling each patient. $61 \%$ from both males and females confirmed wearing gloves only sometimes. $90 \%$ of the female participants always wore gloves before inserting NG tubes where as only $70 \%$ of males did so.

\section{Males who wash their hands before handling each patient}

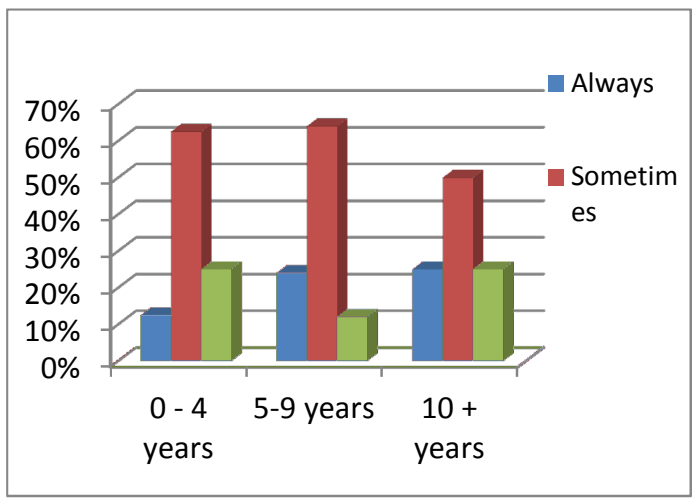

Figure 1 Gender analysis of hand washing

In undertaking a risk assessment regarding safe use of gloves and hand hygiene the most critical factors are availability of gloves, liquid hand wash and antimicrobial hand rub. 59\% confirmed free availability of hand gloves out of which $51 \%$ confirmed of free availability of sterilized hand gloves. About 35\% confirmed unavailability of gloves in the operation theater. 
Availability of Gloves (Sterilized)

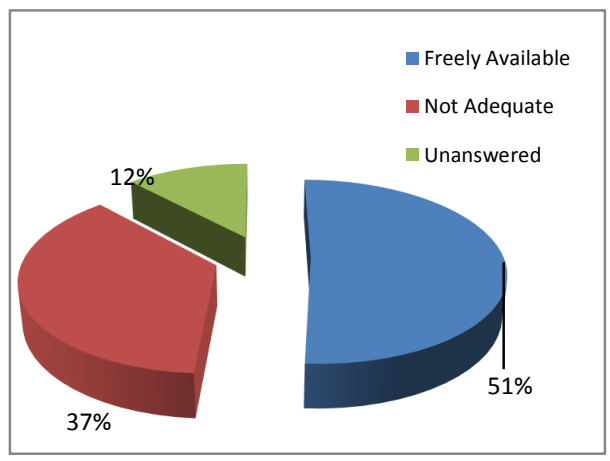

Availability of Gloves (Non sterilized)

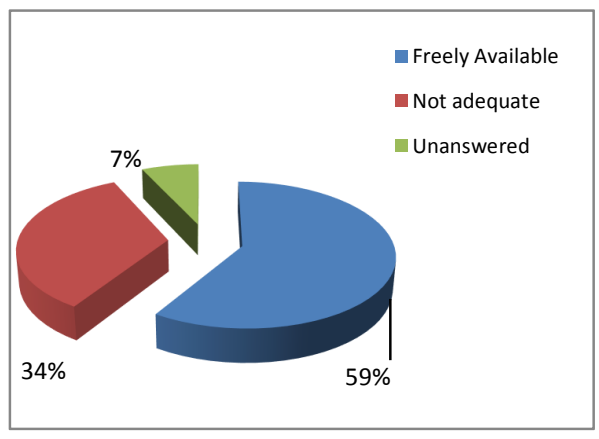

Figure 2 Availability of gloves

While $86 \%$ of the cardiothoracic participants always washed their hands before handling each patient it dropped to $9 \%$ in neurosurgical and $15 \%$ in general surgery participants. $86 \%$ of the cardiothoracic participants used antimicrobial hand rubin between patients as opposed to $4.5 \%$ in neurosurgical and $12 \%$ in general surgery participants. On the contrary it was found that only $6.6 \%$ of the participants wore gloves when performing i.v. cannulation in cardiothoracic practice. $53 \%$ of the cardiothoracic anaesthetists never wore gloves when performing i.v. cannulation. In neurosurgical and general surgery majority didn't wear gloves always and $61 \%$ wore only occasionally.
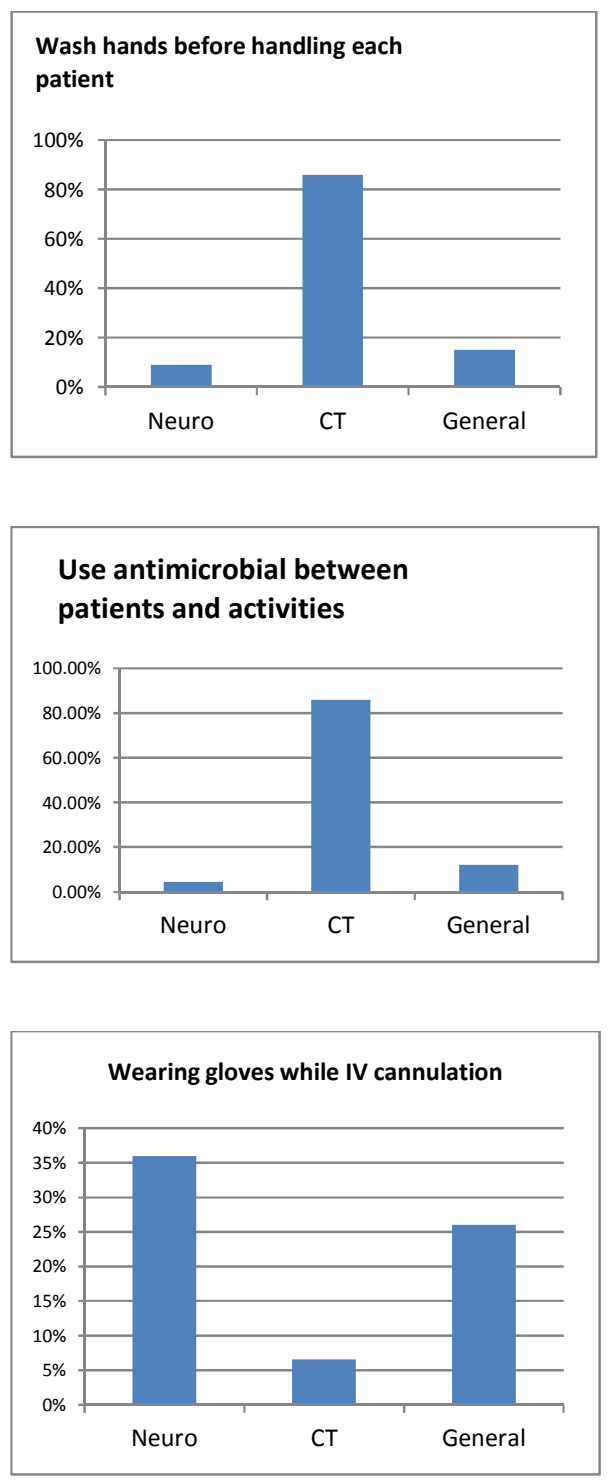

Figure 3 Hand washing and glove wearing practices in different sub specialties (Neuro: Neurosurgical, CT: Cardiothoracic, General : General surgery)

Assessing the frequency of each anaesthetists usage of gloves during each invasive procedure it was noticed that majority wore gloves only sometimes. When inserting NG tubes majority (80\%) confirmed wearing gloves. $2 \%$ did not answer this question and $1 \%$ confirmed not wearing gloves ever while inserting $\mathrm{NG}$ tubes. The extreme scenarios of never using gloves were excluded from the equation. 


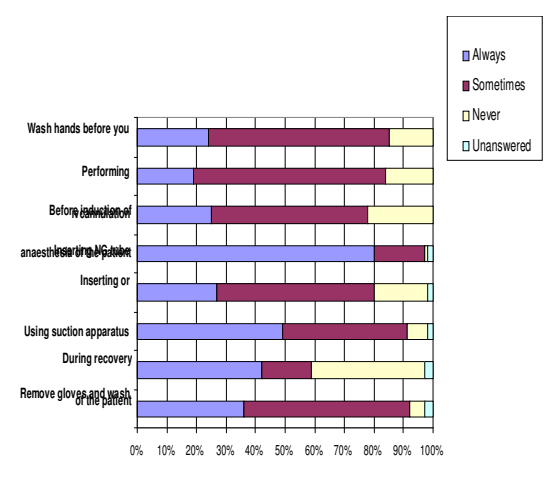

Figure 4 Usage of gloves and hand washing during different procedures

$50 \%$ of the participants who wore gloves before induction of anaesthesia claimed to be using the same gloves while operating the anaesthetic machine possibly leading to easy contamination. $18 \%$ of the participants seemed to be never wearing gloves when inserting oropharyngeal airways. $7 \%$ confirmed that they never use gloves when using suction apparatus where as $49 \%$ always wore gloves and $42 \%$ only sometimes.

As per our study from 100 participants only $25 \%$ always wore gloves before the induction of anaesthesia while $53 \%$ confirmed to be doing it sometimes. $22 \%$ never wore gloves before induction of anaesthesia. $42 \%$ wore gloves during recovery of the patient while $38 \%$ sometimes and $20 \%$ of the participants seem to be not wearing gloves at the recovery stage at all.

$66 \%$ out of those who use gloves at induction removed the before filling the anaesthetic chart and 5\% never removed it. Only 5\% always washed hands before filling the anaesthetic chart and 35\% never washed hands prior to filling the anaesthetic chart. $56 \%$ sometimes washed hands before filling the chart and 4\% did not answer this question.

Irrespective of the situation, in general $72 \%$ of the participants confirmed wearing gloves as important while $27 \%$ thought the importance depended on the situation. $1 \%$ who confirmed it is unimportant to wear gloves was excluded from the analysis.

\section{Discussion}

Infection due to hospital-acquired microbes is an evolving problem worldwide and horizontal transmission of bacterial organisms continues to cause a high nosocomial infection rate in acute care settings. Hands have been implicated as one of the most important transmission sources of nosocomial pathogens in the health care setting. Increased clinical activity has been shown to result in an increase of total bacterial counts on the hands of medical staff $^{3}$. Therefore maintaining good hand hygiene is important prior to patient handling. Further, hand hygiene is considered to be the most simple, inexpensive and effective tool in reducing nosocomial infections. However this study on hand washing compliance, revealed a relatively low compliance rate in given circumstances.

This study illustrates that adherence to hand hygiene best practices during invasive procedures has been extremely low which potentially expose patients and staff to microbial transmission and increases the risk of HCAIs. While adherence was at a poor level it was also highlighted the scarcity of gloves in the operation theatres. Accessibility to hand hygiene supplies is a prerequisite to adequate compliance.

In benchmarking with other similar studies which were done across the world on a similar topic, it was noticed that this study is on par with most of the countries. In an observational study which was done in Karachi, Pakistan with a total 496 observations overall compliance and practice of hand hygiene was found in $62 \%$ ofanaesthetists. It was concluded by identifying that implementation of hand hygiene policy and education has positive effects among anaesthetists. Also it was 
suggested that continuous education and definitive administration measures of hand hygiene practices will improve the situation.

In contrary to above, in a national survey that was done by New Zealand anaesthetists it was found that compliance level is as high as $86 \%$ from 274 participants $^{5}$. It was found that compliance level has increased after the ANZCA guidelines implementation in New Zealand. Further a study done in Great Britain (British Royal Infirmary)in an intensive care unit found that occupational risk of contracting a blood borne viral infection is increased in anaesthetists by not wearing protective gloves for all procedures in which contamination may occur and adherence to the best practises of hand hygiene is not at the required level ${ }^{6}$.

\section{Recommendations and Conclusion}

Over the past two decades, improving patient safety has received growing attention, and one of the first goals of the world health organization's world alliance for patient safety is the substantial reduction of $\mathrm{HAI}^{1}$.To reach this goal, significant improvement in the compliance of healthcare workers (HCWs) with hand hygiene guidelines is considered to be crucial.

Worldwide best practices need to be shared periodically and anaesthetists have a critical role to play in setting best practices of hand hygiene in the theatre. Anaesthetists are frontline workers and are at risk of exposure to potentially serious microorganisms which may not be apparent during the routine care of patients. It is therefore prudent to follow a routine set of precautions aimed at reducing the transmission of microorganisms between patient and anaesthetist or between patients. Hand hygiene should become a routine practice and a gradual culture change should happen within theatres with the guidance of an anaesthetist. Displaying basic best practices in theatres would be another basic step that can be taken to get the message across to the target audience.
Wearing surgical or examination gloves are not sufficient for the prevention of transmission of infections. The relationship between infection control standards and gloving practices is an important issue in combating infectious diseases. Gloves fail frequently, which put health care providers and patients at risk of acquiring fluid borne pathogens. Hence it is also recommended that health supply services evaluate the antimicrobial efficacy of hand rubs and hand wash agents and formulations and specifications of the surgical hand glove preparations.

We need to formulate a proper guideline in infection control in anaesthetic practice which needs to be reviewed and published periodically. By having proper guidelines to follow healthcare organisations will have a legal responsibility to implement changes to reduce healthcare associated infections. Institutional support is crucial to give the message to anaesthetists that good hand hygiene becomes an indispensable part of their clinical culture.

\section{References}

1. WHO. The first Global Patient Safety Challenge: Clean Care is Safer Care, 2005.

2. AAGBI Safety Guideline, Infection Control in Anaesthesia. The Association of Anaesthetists of Great Britain and Ireland. October 2008.

3. Hugonnet S, Perneger TV, Pittet D. Alcohol based hand rub improves compliance with hand hygiene in intensive care units. Arch Intern Med 2002:162:1037-43 http://dx.doi.org/10.1001/archinte.162.9.1037

4. Mueen UK, Khalid MS. Hand washing and gloving practices among anaesthetists. Journal of Pakistan Medical Association 2008:58(1):279

5. Ryan AJ, Webster CS, Merry AF, Grieve DJ. National Survey conducted on Infection control practice by New Zealand Anaesthetists. Anaesthesia and intensive care. 2006;34(1):6874

6. Teare L, Cookson B, Stone S. Hand hygiene. British Medical Journal 2001; 323: 411-12. http://dx.doi.org/10.1136/bmj.323.7310.411 